\title{
Theory overview of BSM top and Higgs interactions
}

\author{
Aldo Deandrea*i \\ Université de Lyon, France; Université Lyon 1, CNRS/IN2P3, UMR5822 IPNL, \\ F-69622 Villeurbanne Cedex, France. \\ E-mail: deandrea@ipnl.in2p3.fr
}

Physics beyond the standard model is actively searched in many experiments and among them the Large Hadron Collider is having at present a central role, exploring a large number of different channels. One of the most interesting and promising sectors for these searches is the one of the top quark, for many different theoretical and phenomenological reasons. In this sector there is a large number of options, from single top, monotop up to multi-top events, all contributing in different ways to clarify and limit the possible structures we may think of beyond the standard model. At the same time the Higgs sector is providing complementary information, both from its detailed properties and in channels associated with the top quark. I shall discuss here only part of this large spectrum of possibilities, in particular suggesting that less standard searches like those on multi-top events, beyond the standard $t-\bar{t}$ and single top searches can bring further insight in constraining and discovering physics beyond the standard model, taking advantage of experimental techniques not so different from those used in present top-quark analyses.

8th International Workshop on Top Quark Physics, TOP2015

14-18 September, 2015

Ischia, Italy

\footnotetext{
* Speaker.

$\dagger$ also Institut Universitaire de France, 103 boulevard Saint-Michel, 75005 Paris, France
} 


\section{Introduction}

Since its discovery in 1995 by the CDF and D0 experiments at the Tevatron [1, 2], the top quark has a special place in the Standard Model (SM) and beyond the standard model (BSM) physics, due to its mass close to the electroweak breaking scale and its peculiar decay properties. One interesting question is whether present searches in top physics, like those for four top final states can be extended to multi-top final states with more tops, and in particular what extra information can be learned. It turns out that even if one can think naively that a very large number of top particles can be produced at the LHC, these multi-top final states in practice have already constraints both regarding the number of particles and the type of production processes. On the other side, scenarios of new physics where a single top quark can be produced, both in association with large missing energy (monotop) or with other particles (single top) offer another handle on BSM physics. All these scenarios have been studied both from the theoretical point of view and by the experimental collaborations. In the following I will consider some of these studies with the particular point of view of looking to the number of top particles in the final state and their implications for top and Higgs physics.

\section{Single- and mono-top physics}

The single top final state is produced through the weak interactions, in processes that allow testing the chiral structure of the production vertex via spin correlations. At the LHC the t-channel amplitude is the dominant one. For example at the $8 \mathrm{TeV} \mathrm{LHC}$ the inclusive production rates of the s-, t- and Wt channels are approximately 5, 85 and 25 picobarns respectively (see [3] for a detailed review of the theoretical calculations and the experimental results), in good agreement with the SM expectations. See also $[4,5,6]$ for the detailed experimental numbers. BSM physics can provide modifications to the SM couplings but also new interactions allowing to produce a single top quark. As an example the decay of a vector-like T' into top quark and $\mathrm{Z}$ boson (see for example the study performed in [7]) gives a typical single top final state from a new flavour changing vertex that is quite common in BSM extensions.

These possibilities can be tested in precision measurement of the top quark properties in the single top final state, but an even more striking BSM signal would be the presence of mono-top events (events in which the top quark is produced alone, with only extra missing energy). The production of such a final state is extremely suppressed in the SM, and also quite peculiar in models of new physics. Two main production mechanisms can lead to a monotop state [8,9]: the resonant production of a coloured bosonic state which decays into a top quark plus an invisible neutral fermion, or via the production of a single top quark in association with a boson that has flavourchanging couplings to top and light quarks and stays invisible in the detector. Embedding the effective couplings of the top quark in an $\mathrm{SU}(2)_{L}$ invariant formalism selects a minimal model for each of the possible production mechanisms [10]. In particular a scalar field coupling with a righthanded top quark and an invisible fermion when the monotop is produced via a resonant channel, and a vector field mediating the interactions of a missing energy particle to right-handed quarks for the non-resonant production mode as discussed in the following. 


\subsection{Resonant monotop production}

\section{Spin-0 mediator}

The initial state consists of a pair of down-type quarks which form a spin-0 state. Since these di-quark states are made of identical quarks and fermions are anticommuting objects, the corresponding wave-functions need to be antisymmetric under the exchange of the quark fields. The exchange of the flavour indices is therefore forced to be antisymmetric too, since the one of the spin and colour indices are antisymmetric and the one of the triplet (adjoint) representation of $\mathrm{SU}(2)_{L}$ is symmetric (for the left-handed quark setup). Therefore the right and left-handed quarks cannot couple to the same scalar and two different objects must thus be introduced. The operators containing the interactions needed for monotop production are

$$
\lambda_{s} \varphi_{s} \bar{d}_{R}^{C} d_{R}+\lambda_{t} \varphi_{t} \bar{q}_{L}^{C} q_{L}+\text { h.c. },
$$

where $q_{L}$ is the left-handed doublet, and $\lambda_{s, t}$ are antisymmetric matrices in flavour space. The second term is the component of a triplet with electric charge $\pm 2 / 3$ that couples eventually to the top. Extra constraints emerge when considering the final state to top-quark plus missing energy due to an invisible fermion $\chi$. Assuming that it is a singlet under the SM symmetries, in order to allow for a $\chi$-coupling to a left-handed top quark, we need to introduce an extra scalar field $\varphi_{d}$ which transforms as a doublet of $S U(2)_{L}$. The operators giving rise to monotop production can then be written as

$$
y_{s} \varphi_{s}^{\dagger} \bar{\chi} t_{R}+y_{d} \varphi_{d}^{\dagger} \bar{\chi} q_{L}+\text { h.c. }
$$

The initial and final state can consequently only be connected via an $S U(2)_{L}$-singlet field $\varphi_{s}$ that couples to right-handed quarks.

\section{Spin-1 mediator}

A similar reasoning can be followed for the case of a spin-1 mediator $X$. The Lorentz vector fermionic bilinear is $\bar{\psi} \gamma_{\mu} \psi$, can be written as $\bar{\psi}_{L} \gamma_{\mu} \psi_{L}+\bar{\psi}_{R} \gamma_{\mu} \psi_{R}$. This implies that the two quarks have the same chirality. In order to build a scalar invariant, vector fields have to couple to these spinors of the same chirality. The couplings of the $X$-field to down-type quarks are

$$
\lambda_{V}^{1} X^{\mu} \bar{d}_{L}^{C} \gamma_{\mu} d_{R}+\text { h.c. }
$$

where the coupling strength is $\lambda_{V}$ and the $X$-boson must be a weak doublet with hypercharge $1 / 6$. The $X$-field has the quantum numbers of a left-handed quark doublet and has two possible options. It can couple to a left-handed top quark and a singlet field $\chi$, but in addition to the interaction relevant for the production of a monotop state, the interaction of a left-handed bottom quark to the second component of the $X$-doublet induces the fast decay of the neutral $\chi$ fermion via an off-shell $X$-state, eliminating the possibility of a monotop signal. The other option consists in considering right-handed quarks. A coupling to right-handed quarks can be obtained if the fermion $\chi$ belongs to an $S U(2)_{L}$ doublet with hypercharge $1 / 2$

$$
\lambda_{V}^{3} X_{\mu} \bar{t}_{R} \gamma^{\mu} \chi_{d}+\text { h.c. }
$$


This model however contains a charged fermion that can be produced in association with a top via the vector of charge $-1 / 3$ and is therefore likely to be constrained by channels different from the monotop one.

The previous analysis allows to conclude that the minimal and natural choice for the resonant monotop channel is the spin- 0 mediator with a new fermion both gauge-singlet and coupling to right-handed quarks. The couplings of the scalar to the down quarks are antisymmetric under the exchange of the flavour indices. Consequently, parton density effects enhance the production mode $\bar{d} \bar{s} \rightarrow \varphi$ at hadron colliders such as the LHC.

\subsection{Non-resonant monotop production}

In this scenario the top quark is produced in association with an invisible bosonic field that couples in a flavour-changing way to top and light up-type (up or charm) quarks. The bosonic state is in general not stable since it couples to quarks and therefore the missing energy signature is enforced by requiring these fields either to be long-lived or to decay predominantly into a pair of additional neutral stable particles.

\section{Spin-0 mediator}

The coupling in the effective Lagrangian is

$$
y^{i j} \phi \bar{q}_{L, i} u_{R, j}+\text { h.c. }
$$

where $i, j$ are the quark flavour indices. Gauge invariance implies the presence of interactions between the charged component field $\phi^{+}$and quarks, so that the $\phi^{+}$field decays always promptly into two-body final states, $\phi^{+} \rightarrow u \bar{b}$ or $t \bar{d}$. Analogously, the neutral component $\phi^{0}$ could also decay into an associated particle pair comprised of a top and an up quark, $\phi^{0} \rightarrow u \bar{t}+t \bar{u}$, as well as into a three-body final state via the exchange of a virtual charged scalar field.

If we restrict ourselves to $\phi^{0}$-decays into fermionic particles, the minimal option is given by the Lagrangian

$$
\mathscr{L}_{\phi-\text { decay }}=y_{\chi} \phi \bar{\chi}_{d} \chi_{s}+\text { h.c. },
$$

where $\chi_{s}$ is an electroweak singlet and $\chi_{d}$ a weak doublet with hypercharge of $1 / 2$. As a consequence of this non-minimal dark matter sector of the model, monotop production via flavourchanging interactions of up-type quarks with a new invisible scalar field will always be accompanied by an extra single top production mode

$$
p p \rightarrow t \phi^{-} \rightarrow t \chi_{d}^{0} \chi_{s}^{0}\left[W^{-}\right]^{*} .
$$

The nature and magnitude of the associated effects are very model dependent.

\section{Spin-1 mediator}

If the mediator is a vector boson $V$, one can build very simple models, since it can be singlet under the electroweak group. The associated couplings involve either right-handed or left-handed quarks and take the form

$$
\left(a_{R}^{i j} V_{\mu} \bar{u}_{R, i} \gamma^{\mu} u_{R, j}+a_{L}^{i j} V_{\mu}\left(\bar{u}_{L, i} \gamma^{\mu} u_{L, j}+\bar{d}_{L, i} \gamma^{\mu} d_{L, j}\right)+\text { h.c. }\right)
$$


where the $a_{L, R}$ parameters denote the strengths of the interactions of the $V$-field with the quarks. A monotop signal is expected only when $V$ is invisible and dominantly decays into a pair of dark matter particles.
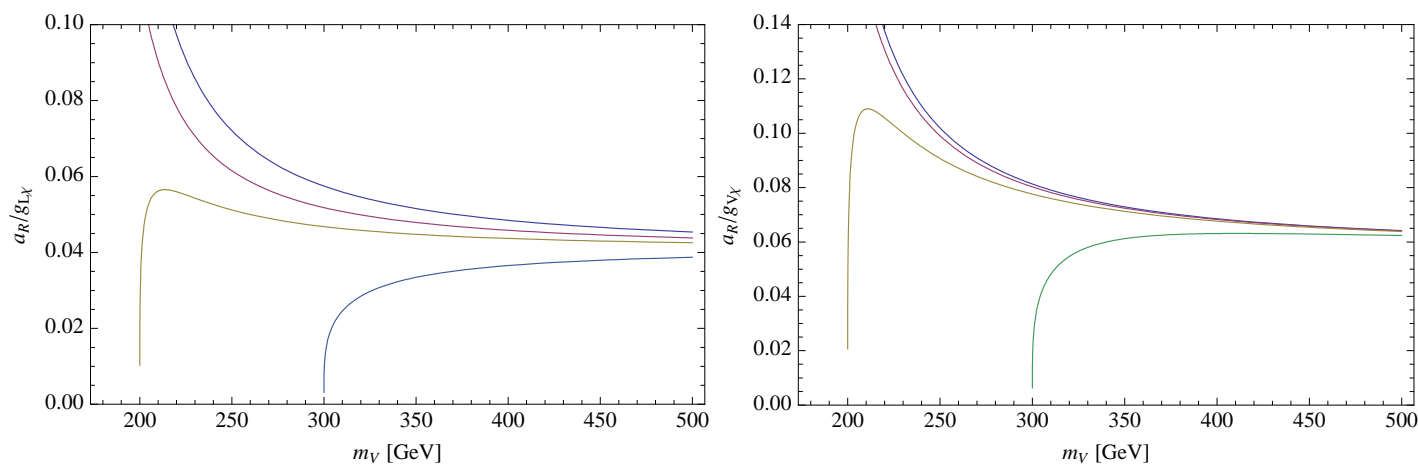

Figure 1: Maximum value of $a_{R}$ necessary to enforce the mediator $V$ to decay invisibly in $99 \%$ of the cases. We focus on scenarios where the couplings of the mediator to dark matter are chiral with $g_{R \chi}=0$ (or $g_{L \chi}=0$ ) in the left panel, and vector with $g_{L \chi}=g_{R \chi}=g_{V \chi}$ in the right panel. The four curves correspond to $m_{\chi}=5,75,100$ and $150 \mathrm{GeV}$ from the lower to the upper ones in each figure.

Searches limiting the parameter space of these minimal models (both resonant and non-resonant) have been performed by ATLAS and CMS [11,12]. Earlier searches for dark matter production in the mono-jet or single top quark plus missing transverse energy signature were performed by CDF $[13,14]$.

\section{Multi-top physics}

Several different models can give rise to the final states with many top quarks in the final state and here we consider models based on the assumption that new physics couples mainly to the top quark (such as a "top portal" kind of scenario). The topologies we consider, consist of the decay chains of pair-produced coloured particles, which will either be "coloron"-like bosons (see for example [15]) or $T^{\prime}$ vector-like fermions (see for example [16, 17]).

Note that no matter how complicated the decay chain is, there will be only one parameter which strongly influences the event yield: the mass of the pair-produced particles. Therefore limits and observation windows estimated using cuts and simple event counting with these topologies are generic and can be generalised to other models with similar final states.

\subsection{Four and six tops}

Four top production occurs in the Standard Model through a big set of diagrams, with production dominated by gluon-initiated diagrams. Its sensitivity to BSM physics, with sometimes huge enhancement in the cross section with respect to the SM, is the main reason for the large interest in this final state, which allows to test both resonant and non resonant BSM physics. For example contact interactions of a composite top quark would have consequences not only on top pair production but also on the four top final state [18, 19, 20]. The four top final state in channels including explicitly new BSM particles is also extensively studied in the literature (see [21, 22, 23, 24] for 


\begin{tabular}{|c|c|c|}
\hline & $R_{Z^{\prime}}$ & $R_{T}$ \\
\hline$R_{1}$ & $\mathbf{1}$ & $\mathbf{3}$ \\
\hline$R_{2}$ & $\mathbf{8}$ & $\mathbf{3}$ \\
\hline$R_{3}$ & $\mathbf{8}$ & $\overline{\mathbf{6}}$ \\
\hline$R_{4}$ & $\mathbf{8}$ & $\mathbf{1 5}$ \\
\hline
\end{tabular}

Table 1: Possible colour assignments for $T$ and $Z^{\prime}$ in the 6-top topology.

few examples), and searched in detail by the LHC experimental collaborations [25, 26]. Typically the four top quarks are the result of the decay of "heavy gluon" octet or of a coloured singlet which is pair produced and decaying to $t \bar{t} t \bar{t}$. More recently, in the framework of composite models also the case of a colour sextet pair produced and decaying to $t t \bar{t} \bar{t}$ was considered [27]. As the ttitt $\bar{t}$ final state is well covered in the existing literature I will not enter into more details here.

We can therefore consider in more detail the six top quark final state, by analysing the production and decay of such a process together with simple analyses which can be used to bound it. Having a six top final state requires a top partner $T$ and a bosonic particle, which is typically a $Z^{\prime}$. A reasonable choice for the gauge group is $(\mathbf{3}, \mathbf{1}, 2 / 3)$ for $T$ and $(\mathbf{1}, \mathbf{1}, 0)$ for $Z^{\prime}$. More exotic assignments are possible, as listed in table 1 , but having an additional coloured particle leads to a typically more constrained model. Typical mass limits for a top partner $T$ are close to the $1 \mathrm{TeV}$ range from present LHC searches. Present bounds on the sixâĂŞtop final state come mainly from analyses including two and three same sign leptons, which have a very reduced background. The CMS paper on same-sign di-leptons production associated with b-jets (2SSL+b) [29] is sensitive to the 6-top final states, so it is possible to recast it in our case to set limits on the 6-top (or more) parameter space. Other analyses which are sensitive to multi-lepton, multi-jet, İÿET final states, are ATLAS supersymmetry searches [28, 30].

Performed a parameter scan in the plane $M_{T}, M_{Z^{\prime}}$ in the minimal colour embedding model, an exclusion region below $M_{T}<710 \mathrm{GeV}$, as shown in Figure 2 can be obtained recasting the CMS analysis [29]. This limit has mild dependance on $M_{Z^{\prime}}$. It can be extrapolated to more unusual colour structures of Table 1 by multiplication of the signal yield by the corresponding colour factor (neglecting colour correlation effects for simplicity).

\subsection{Eight tops}

One can build on the previous case in order to have a decay chain producing eight top quarks, by adding an extra coloured boson $\rho$ decaying to $t \bar{T}$. The $\rho$ can be pair produced in $p p$ collisions by QCD. The six-top final state can also arise and all the above discussion applies. The colour assignments for $T$ and $Z^{\prime}$ are constrained as discussed in the previous sections, which imposes that the $\rho$ is a colour-octet in the minimal choice for $T$ and $Z^{\prime}$ quantum numbers.

The best way to detect a multi-top final state is through leptonic channels, based on the result of existing searches and exploiting the possibility of having same-sign leptons allows to strongly reduce Standard Model backgrounds. Performing a Monte-Carlo simulation one can check that, as expected, the $H_{T}$ spectrum is harder for eight-top events. The $E_{T}$ and leptonic $p_{T}$ are slightly lower than in the six-top case, since individual tops have lower momentum. These results limit strongly the possibility to put bounds on $M_{\rho}$ with the $2 \mathrm{SSL}+\mathrm{b}$ analysis using Run 1 data. In particular the 


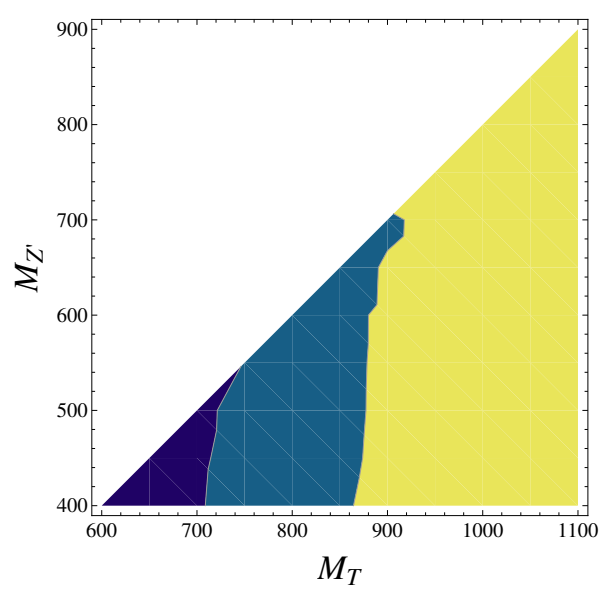

Figure 2: Limits in the plane $\left(M_{T}, M_{Z^{\prime}}\right)$ from the recasting of the CMS analysis. Purple area is excluded at $2 \sigma$, blue area at $1 \sigma$. Yellow area is not excluded.

results of the CMS analysis is compatible with the background hypothesis within less than $1 \sigma$. The 3SSL analysis is not constraining either as even though combinatorics may help reducing the signal loss, no signal is expected at the LHC with the amount of data available from Run 1 . The ongoing Run 2 can bring however the possibility to increase the limits on top multiplicity as much stronger bounds should be placed on six-top processes, and eight top processes should start to become visible. Further experimental scrutiny in this direction is therefore a unique tool to constrain or discover BSM effects in the top sector.

\section{Acknowledgments}

I wish to thank my collaborators G.Cacciapaglia, N.Deutschmann, B. Fuks, and also T. TheveneauxPelzer, J. Donini and F. Maltoni for useful discussions over many of the topics discussed here. I acknowledge support by the Theory-LHC France initiative of CNRS/IN2P3; the Labex-LIO (Lyon Institute of Origins) under grant ANR-10-LABX-66 and FRAMA (FR3127, Fédération de Recherche "André Marie Ampère").

\section{References}

[1] F. Abe et al. [CDF Collaboration], Phys. Rev. Lett. 74 (1995) 2626 [hep-ex/9503002].

[2] S. Abachi et al. [D0 Collaboration], Phys. Rev. Lett. 74 (1995) 2632 [hep-ex/9503003].

[3] A. Giammanco, Rev. Phys. 1 (2016) 1 [arXiv:1511.06748 [hep-ex]].

[4] ATLAS Collaboration, ATLAS-CONF-2015-047.

[5] ATLAS and CMS Collaborations, ATLAS-CONF-2013-098, http://cds.cern.ch/record/1601029?ln=en

[6] G. Aad et al. [ATLAS Collaboration], arXiv:1510.03752 [hep-ex] and CMS-PAS-TOP-14-009.

[7] L. Basso and J. Andrea, JHEP 1502 (2015) 032 [arXiv:1411.7587 [hep-ph]].

[8] J. Andrea, B. Fuks and F. Maltoni, Phys. Rev. D 84 (2011) 074025 [arXiv:1106.6199 [hep-ph]]. 
[9] J. L. Agram, J. Andrea, M. Buttignol, E. Conte and B. Fuks, Phys. Rev. D 89 (2014) 1, 014028 [arXiv:1311.6478 [hep-ph]].

[10] I. Boucheneb, G. Cacciapaglia, A. Deandrea and B. Fuks, JHEP 1501 (2015) 017 [arXiv:1407.7529 [hep-ph]].

[11] G. Aad et al. [ATLAS Collaboration], Eur. Phys. J. C 75 (2015) 2, 79 [arXiv:1410.5404 [hep-ex]].

[12] V. Khachatryan et al. [CMS Collaboration], Phys. Rev. Lett. 114 (2015) 10, 101801 [arXiv:1410.1149 [hep-ex]].

[13] T. Aaltonen et al. [CDF Collaboration], Phys. Rev. Lett. 108 (2012) 211804 [arXiv:1203.0742 [hep-ex]].

[14] T. Aaltonen et al. [CDF Collaboration], Phys. Rev. Lett. 108 (2012) 201802 [arXiv:1202.5653 [hep-ex]].

[15] E. H. Simmons, A. Atre, R. S. Chivukula, P. Ittisamai, N. Vignaroli, A. Farzinnia and R. Foadi, [arXiv:1304.0255 [hep-ph]].

[16] Y. Okada and L. Panizzi, Adv. High Energy Phys. 2013 (2013) 364936 [arXiv:1207.5607 [hep-ph]].

[17] M. Buchkremer, G. Cacciapaglia, A. Deandrea and L. Panizzi, Nucl. Phys. B 876 (2013) 376 [arXiv:1305.4172 [hep-ph]].

[18] B. Lillie, J. Shu and T. M. P. Tait, JHEP 0804 (2008) 087 [arXiv:0712.3057 [hep-ph]].

[19] A. Pomarol and J. Serra, Phys. Rev. D 78 (2008) 074026 [arXiv:0806.3247 [hep-ph]].

[20] K. Kumar, T. M. P. Tait and R. Vega-Morales, JHEP 0905 (2009) 022 [arXiv:0901.3808 [hep-ph]].

[21] M. Battaglia and G. Servant, Nuovo Cim. C 033N2 (2010) 203 [arXiv:1005.4632 [hep-ph]].

[22] T. Gregoire, E. Katz and V. Sanz, Phys. Rev. D 85 (2012) 055024 [arXiv:1101.1294 [hep-ph]].

[23] G. Cacciapaglia, R. Chierici, A. Deandrea, L. Panizzi, S. Perries and S. Tosi, JHEP 1110 (2011) 042 [arXiv:1107.4616 [hep-ph]].

[24] J. A. Aguilar-Saavedra and J. Santiago, Phys. Rev. D 85 (2012) 034021 [arXiv:1112.3778 [hep-ph]].

[25] ATLAS Collaboration, "Search for exotic same-sign dilepton signatures (b' quark, $T_{5 / 3}$ and four top quarks production) in $4.7 / \mathrm{fb}$ of pp collisions at $\sqrt{s}=7 \mathrm{TeV}$ with the ATLAS detector," [ATLAS-CONF-2012-130].

[26] CMS Collaboration, "Search for standard model four top quark production at $8 \mathrm{TeV}$ in the lepton + jets channel," [CMS-PAS-TOP-13-012].

[27] G. Cacciapaglia, H. Cai, A. Deandrea, T. Flacke, S. J. Lee and A. Parolini, JHEP 1511 (2015) 201 [arXiv:1507.02283 [hep-ph]].

[28] G. Aad et al. [ATLAS Collaboration], JHEP 1406 (2014) 124 [arXiv:1403.4853 [hep-ex]].

[29] S. Chatrchyan et al. [CMS Collaboration], JHEP 1303 (2013) 037 [JHEP 1307 (2013) 041] [arXiv:1212.6194 [hep-ex]].

[30] G. Aad et al. [ATLAS Collaboration], JHEP 1406 (2014) 035 doi:10.1007/JHEP06(2014)035 [arXiv:1404.2500 [hep-ex]]. 